\title{
Exploration du vécu de l'usure de compassion chez les infirmières œuvrant en oncologie clinique
}

\author{
par Beth Perry, Greg Toffner, Trish Merrick et Janice Dalton
}

\section{Abrégé}

L'usure de compassion (UC) est "une fatigue débilitante provoquée par des réactions empathiques répétées à la douleur et à la souffrance d'autrui» (LaRowe, 2005, p. 21, traduction libre). Les tâches accomplies par les infirmières en oncologie et le vécu des personnes qu'elles soignent les exposent à un risque élevé d'UC (Pierce et al., 2007; Ferrell \& Coyle, 2008). C'est la raison pour laquelle l'étude porte particulièrement sur leur cas. Cet article réalise un survol détaillé d'une étude de recherche qualitative d'ordre descriptif et exploratoire qui s'intéressait au vécu de l'UC chez les infirmières canadiennes ouvrant en oncologie clinique. Le cadre de l'étude s'inspire d'un modèle conceptuel du processus de stress d'Aneshensel, Perlin, Mullan, Zarit et Whitlatch (1995) lequel examine le stress des soignants dans quatre domaines (voir la figure 1). Dix-neuf participantes ont été recrutées pour l'étude par le biais d'une publicité publiée dans la Revue canadienne de soins infirmiers en oncologie (RCSIO). Celle-ci dirigeait les participants éventuels vers un site Web universitaire conçu spécialement pour l'étude. Les participants remplissaient un questionnaire, rédigeaient un récit décrivant une de leurs expériences de l'UC et envoyaient le tout au site Web de recherche sécurisé. Les données ont fait l'objet d'une analyse thématique. Cina thèmes ont été cernés: définition de l'UC, causes de l'UC, facteurs aggravant l'UC, facteurs atténuant l'UC et enfin, conséquences de l'UC. L'UC pouvait être déclenchée par les connaissances limitées des participants à son sujet, le manque de soutien externe et le manque de temps pour dispenser des soins de qualité. L'écart entre les soins de qualité que les infirmières souhaitaient prodiguer et les soins qu'elles dispensaient aggravait l'UC, lorsque s'y ajoutaient du stress physique et/ou émotionnel coexistant. Par contre, l'UC était atténuée par le soutien des collègues, un juste équilibre travail-vie personnelle, des liens avec autrui, la reconnaissance d'autrui, la maturité et l'expérience. Les conséquences de l'UC comprenaient une profonde fatigue psychique et physique, des effets négatifs sur les relations personnelles et l'envie de quitter la spécialité. L'article présente des recommandations visant à améliorer le bien-être des infirmières en oncologie.

\section{Introduction}

Le cancer est une maladie grave qui menace le pronostic vital et s'accompagne souvent de souffrances physiques, émotionnelles et/ ou spirituelles. Ce sont les infirmières en oncologie qui assument en majeure partie la responsabilité de la fourniture des soins à ces

\section{Au sujet des auteurs}

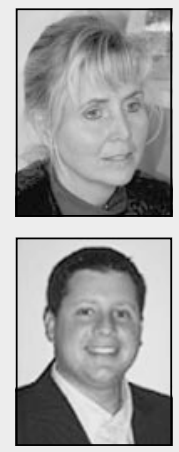

Beth Perry, inf., Ph.D., Professeure agrégée, Center for Nursing and Health Studies, Universite Athabasca, 8708-116 Ave., Edmonton, AB T5B 0M6. Téléphone (bureau) : 1-866-804-7721; Télécopieur : 780-675-6468; Courriel : bethp@athabascau.ca

Greg Toffner, B.Sc., MRT(R), RTR, M.Sc.S. (candidat)

Trish Merrick, inf., B.Sc.inf.

Janice Dalton, RD, M.Sc.S. malades. La complexité des soins exigés, de concert avec l'intensité potentiellement forte de la relation infirmière-patient dans les contextes oncologiques, peut exposer les infirmières à un risque élevé d'UC et c'est la raison pour laquelle elles sont le sujet de cette étude. Cependant, malgré ce risque important, l'UC demeure un phénomène fort peu exploré chez ce groupe de soignants (Bush, 2009). Cette étude de recherche qualitative de nature descriptive et exploratoire a examiné le vécu de l'UC chez les infirmières canadiennes œuvrant en oncologie clinique. Les données recueillies ont permis de dégager la définition que les infirmières en oncologie donnent de l'UC, les facteurs qui provoquent, aggravent et/ou atténuent l'UC ainsi que les conséquences de l'UC parmi ce groupe de professionnels. Un modèle conceptuel examinant le processus de stress des soignants dans quatre domaines fournit le cadre d'analyse et étaye les recommandations (Aneshensel et al., 1995) (voir la figure 1). Cette étude est importante puisqu'elle explore un phénomène qui peut avoir une incidence négative directe sur le bien-être des infirmières en oncologie ainsi qu'une incidence négative indirecte sur les personnes auxquelles elles fournissent des soins. Les infirmières qui ne jouissent pas d'une santé optimale sont dans l'incapacité de dispenser des soins de qualité (Perry, 2009). Une meilleure compréhension de l'UC chez les infirmières en oncologie est le premier pas vers l'élaboration et la mise en œuvre de stratégies visant à prévenir ou à minimiser l'UC chez ce groupe de soignants.

\section{Revue de la littérature}

Vers une définition de l'usure de compassion. Le terme "compassion fatigue» [= usure de compassion] a été utilisé pour la première fois en 1992 par Joinson pour décrire les stresseurs particuliers touchant les personnes qui œuvrent dans le secteur des soins de santé. LaRowe (2005) a décrit l'UC comme étant «une fatigue débilitante provoquée par des réactions empathiques répétées à la douleur et à la souffrance d'autrui » (p. 21, traduction libre). Bush (2009) ajoute que l'UC est un état émotionnel présentant des conséquences psychologiques et physiques qui provient de la fourniture de soins aigus ou prolongés à des personnes aux prises avec des événements traumatiques, des souffrances ou des malheurs de grande intensité.

Quoique le terme soit bien défini, il règne encore une certaine confusion conceptuelle à son égard. L'UC est un des nombreux termes employés afin de décrire les effets négatifs de la dispensation de soins aux personnes éprouvant des traumatismes (Abendroth \&

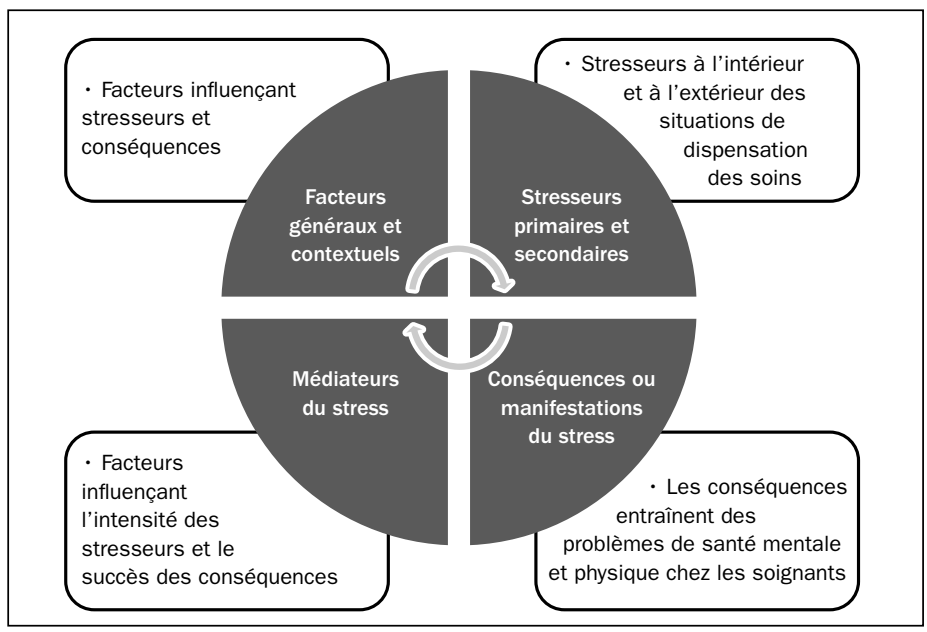

Figure 1. Modèle du processus de stress

Adapté d'Aneshensel, Pearlin, Mullan, Zarit et Whitlatch, 1995. 
Flannery, 2006). Les termes suivants sont parfois employés à titre de synonymes de l'UC : fatigue de compassion, traumatisation secondaire ou traumatisme transmis par personne interposée, stress vicariant, état de stress post-traumatique, détresse morale et épuisement professionnel (Ferrell \& Coyle, 2008; Munroe, 1995; Sabo, 2006; Davis, 1996). L'élément commun à tous ces syndromes, y compris l'UC, est l'accent mis sur le lourd tribut physique et psychologique que paient les soignants du fait de la fourniture de soins. Tout le monde s'entend pour dire que quelque soit le nom qu'on lui donne, le travail auprès des personnes éprouvant des souffrances et des maladies à issue potentiellement mortelle peut se solder par des conséquences nocives pour les soignants (Abendroth \& Flannery, 2006).

Cependant, l'UC diffère des autres syndromes ci-dessus pouvant toucher les soignants (Bush, 2009). Par exemple, dans le cas de l'état de stress post-traumatique, le soignant est exposé à un ou plusieurs événement(s) traumatisant(s) alors que l'UC est l'exposition à une personne traumatisée et qu'il se produit une absorption subconsciente du traumatisme vécu par cette dernière (Sabo, 2006). De plus, l'UC et l'épuisement professionnel ne sont pas identiques bien qu'aux dires des spécialistes, la présence de l'épuisement professionnel est susceptible d'augmenter la probabilité de survenue de l'UC (Sabo). Ils voient dans l'UC une conséquence «naturelle» de la dispensation des soins aux personnes qui souffrent, tandis que l'épuisement professionnel est une réaction à des déclencheurs négatifs au sein de l'environnement de travail comme la charge de travail et l'incapacité à atteindre les objectifs de travail (Sabo). Alors que l'épuisement professionnel met en jeu une usure progressive du soignant, l'apparition de l'UC est souvent bien plus abrupte (Magraith, Benson \& Yunti, 2005). D'aucuns pensent qu'il est possible de traiter l'épuisement professionnel en éloignant les soignants concernés des déclencheurs liés au lieu de travail tandis que l'UC peut avoir des conséquences négatives plus durables pour le soignant et qu'il n'existe pas de traitement évident contre elle (Sabo, 2006). La détresse morale se rapporte aux défis éthiques éprouvés par les professionnels de la santé lorsqu'ils soignent des patients gravement malades et savent ce qu'il faudrait faire mais ne le font pas (Davis, 2006). Alors que l'UC peut être exacerbée par la détresse morale, l'UC est perçue comme étant une expérience distincte (Sabo, 2006). Les infirmières jugent que l'UC reflète le mieux l'expérience qu'elles vivent dans leur travail auprès de personnes éprouvant de grandes souffrances (Sabo, 2006). Tout ce qui précède explique pourquoi l'UC constitue le sujet de la présente étude.

Les équipes de recherche ont conclu que l'UC survient dans un éventail de professions de la santé notamment les suivantes : travail social, recherche clinique, ergothérapie et physiothérapie, consultation génétique et médecine (Adams, Figley \& Bascarino, 2008; Badger, Royse \& Craig, 2008; Pickett, Brennan, Greenberg et al., 1994; Stebnicki, 2002; Costa, 2005; Benoit, McCarthy \& LeRoy, 2007; Pfifferling \& Gilley, 1999). Les études indiquent que les infirmières sont particulièrement susceptibles d'être touchées par l'UC étant donné que la compassion et l'empathie font partie des valeurs fondamentales auxquelles elles ont recours pour effectuer leur travail (Figley, 1995). En faisant appel à ces valeurs, les infirmières en oncologie se mettent dans une situation où elles risquent de «subir des blessures au travail» (Stebnicki, 2002). On peut voir dans l'empathie une arme à double tranchant, c'est-à-dire une valeur fondamentale essentielle à la réussite de l'infirmière, d'une part, et un point de vulnérabilité, d'autre part (Larson, 1993). Il est donc possible que l'esprit de compassion qui amène les infirmières à embrasser cette carrière les rende également vulnérables à l’UC (Bush, 2009).

Quoique les infirmières en oncologie courent un risque particulièrement élevé d'éprouver l'UC, fort peu d'études ont été consacrées à ce problème au sein de leur profession (Bush, 2009; McMullen, 2007). De plus, les études existantes sur l'UC chez les infirmières en oncologie sont toutes de nature préliminaire. Par exemple, Pierce et ses collaborateurs (2007) ont conclu que les infirmières en oncologie ne connaissent pas bien le terme «usure de compassion» ni les symptômes afférents. Absolon et Krueger (2008) ont étudié l'efficacité d'un groupe de soutien lié à l'UC au sein d'un groupe d'infirmières en gynéco-oncologie. En se fondant sur des résultats de recherche restreints, Aycock et Boyle (2008) ont suggéré diverses interventions permettant de gérer l'UC chez les infirmières en oncologie. Pour résumer, la nature de leur travail et le vécu des patients qu'elles soignent exposent les infirmières en cancérologie à un risque élevé d'UC. Et pourtant, on constate une pénurie de travaux de recherche qui permettraient de mieux comprendre le vécu de l'UC parmi ce groupe professionnel (Ferrell \& Coyle, 2008).

Conséquences de l'UC en matière de santé. Seules quelques études ont exploré les conséquences de santé éventuelles, chez les infirmières, de leurs attitudes de compassion (Abendroth \& Flannery, 2006; Sabo, 2008). Les explorations actuelles semblent indiquer que l'UC pourrait avoir une profonde incidence négative sur la santé mentale et physique des soignants (Sabo, 2008; Figley, 1995). Les effets de l'UC peuvent survenir selon un continuum allant d'aigu à chronique et touchant les sept domaines ci-après : cognitif, émotionnel, comportemental, relations personnelles, somatique, rendement au travail et spirituel (Figley, 1995). Les symptômes cognitifs incluent entre autres la perte de concentration, la faible estime de soi et l'apathie (Maslach, Jackson \& Leiter, 1996). Les symptômes d'ordre émotionnel comprennent entre autres l'angoisse, la culpabilité, la dépression, la colère et le sentiment d'impuissance (Bush, 2008). Les symptômes d'ordre comportemental sont notamment l'irritabilité, les modifications de l'appétit et la fatigue (Maslach et al., 1996). L'UC perturbe les relations personnelles à cause de la méfiance envers autrui et du comportement de retrait (Bush, 2009). Les maux de nature somatique vont d'une douleur généralisée à l'immunité compromise (Maslach et al., 1996). Les infirmières peuvent éprouver des symptômes d'ordre spirituel à cause de l'UC tels que la remise en question de la signification et du but de la vie et des doutes visà-vis de leurs croyances et valeurs personnelles (Bush, 2009). En somme, il est possible que les infirmières qui éprouvent l'UC soient incapables de travailler efficacement et qu'elles subissent en permanence des changements malsains dans la manière dont elles perçoivent leur propre personne, les autres et leur travail (Bush, 2009). En outre, les incidences cumulatives de l'UC peuvent nuire au bien-être personnel et professionnel des infirmières, entraîner un profond épuisement émotionnel et physique et les amener à se questionner sur la valeur du travail qu'elles effectuent (Robinson, Clements \& Land, 2003).

\section{Méthodologie}

Cette étude qualitative de nature descriptive et exploratoire s'intéressait au vécu de l'UC chez les infirmières canadiennes en oncologie clinique. Elle visait à explorer la façon dont ces infirmières décrivaient leur vécu de l'UC. Les résultats de l'étude pourraient éclairer de nouveaux projets de recherche sur le sujet. L'objet de l'étude a été raffiné afin d'inclure les facteurs qui causent, aggravent et/ou atténuent l'UC chez les infirmières œuvrant en oncologie clinique. La question de recherche posée était «Comment les infirmières œuvrant en oncologie clinique décrivent-elles leur vécu de l'UC?»

Cadre conceptuel. Le modèle conceptuel retenu pour cette étude, intitulé modèle du processus de stress, examine le stress des soignants dans les quatre domaines suivants : les antécédents et le contexte en matière de stress; les stresseurs; les médiateurs du stress; et enfin, les conséquences ou les manifestations du stress (Aneshensel et al., 1995). Ce modèle précise de multiples aspects du stress des soignants y compris des stresseurs primaires émanant directement des interactions avec les patients ainsi que des stresseurs secondaires dus à des circonstances externes à la situation de prestation des soins. Le modèle suggère que les antécédents 
et le contexte influencent les stresseurs et les conséquences. On pense que les conditions de médiation exercent une influence sur l'intensité des stresseurs et sur le degré de succès des conséquences et que ces conditions ont une incidence sur la relation entre les stresseurs et les conséquences. Pour résumer, les facteurs liés aux antécédents et au contexte, les stresseurs et les médiateurs interagissent pour produire des conséquences lesquelles débouchent sur des problèmes de santé physique et mentale chez les soignants (voir la figure 1).

Échantillonnage et recrutement. Avant de procéder au recrutement des participants, l'approbation éthique de l'étude a été obtenue auprès du comité d'éthique de la recherche de l'Université Athabasca (AUREB). Les participants à l'étude étaient des infirmières en oncologie clinique qui répondaient à la définition selon laquelle elles possédaient une formation étendue en soins en cancérologie et consacraient la majorité de leur temps à dispenser des soins à des patients atteints de cancer (ACIO, n.d.). Dix-neuf participantes ont ainsi été recrutées à l'échelle du Canada grâce à une publicité placée dans la RCSIO. Les critères d'inclusion exigeaient notamment que les participants occupent actuellement un poste d'infirmière œuvrant en oncologie clinique, aient éprouvé l'UC telle que définie par LaRowe (2005), aient accès à Internet et puissent communiquer en anglais ou en français. Un résumé des données démographiques relatives aux participantes est présenté au tableau 1.

Collecte des données. Les participantes éventuelles ont répondu à une publicité de recrutement d'envergure nationale en se rendant dans le site Web sécurisé de l'équipe de recherche mis spécifiquement en place pour cette étude; elles avaient la possibilité de consentir à participer à l'étude, de remplir un questionnaire en ligne et de rédiger un récit décrivant un épisode d'UC particulier. Toutes les données ont ensuite été envoyées de manière anonyme à la chercheuse principale par l'intermédiaire du serveur de l'Université Athabasca qui a supprimé les adresses IP des candidates à la participation. Les données (brutes et analysées) ont été conservées dans des ordinateurs protégés par des mots de passe et dans des systèmes de secours verrouillés et ont été détruites (au moyen de Shred 2) par la chercheuse principale une fois terminée l'analyse des données. Toutes les données ont été manipulées en conformité avec les lignes directrices déontologiques stipulées par l'AUREB.

Analyse des données. L'équipe de recherche incluait la chercheuse principale qui a une expertise des soins infirmiers en oncologie et de l'UC, une étudiante de maîtrise en sciences infirmières possédant une expertise naissante de l'analyse de données de nature qualitative et des infirmières en oncologie œuvrant en milieu clinique. Les données ont été lues et relues individuellement par chacun des membres de l'équipe de recherche. Les énoncés importants et leurs significations possibles ont été dégagés séparément par chaque membre de l'équipe. Durant cet exercice de lecture initial, les chercheurs se sont efforcés d'ignorer leurs présuppositions sur les phénomènes afin que ceux-ci soient perçus tels qu'ils sont et non pas tels qu'ils paraissent à la lumière des idées préconçues (Oiler, 1986). Après avoir examiné les données chacun de leur côté, les membres de l'équipe ont travaillé ensemble à l'organisation des significations en thèmes et à l'intégration des résultats au sein d'une description préliminaire du phénomène (Oiler). Les chercheurs étaient unanimes quant aux thèmes, et dans les rares cas où ils n'étaient pas immédiatement du même avis, ils faisaient appel au dialogue et à la discussion continue pour parvenir au consensus. Les données ont été gérées à l'aide du logiciel Word.

Légitimité et limites des données. Afin de maximiser la légitimité des données (crédibilité et validité), les commentaires des infirmières sont présentés mot à mot (Lincoln \& Guba, 1985). L'échantillon a fourni une population variée (à l'intérieur des critères d'inclusion stipulés). Cela accroît la crédibilité des données puisqu'on obtient une description approfondie du phénomène à l'étude grâce aux multiples points de vue considérés (Loiselle \& Profetto-McGrath, 2004). La crédibilité de la chercheuse principale constitue également une composante de la légitimité des données étant donné que, dans les études qualitatives, elle est l'instrument de collecte et d'analyse des données (Loiselle \& Profetto-McGrath). La chercheuse principale a une vaste expérience de la réalisation d'études qualitatives sur Internet. Afin d'assurer la confirmabilité, la chercheuse principale et ses assistants ont examiné indépendamment toutes les sources de données, puis se sont consultés les uns les autres pour poursuivre l'analyse. Ceci a permis d'assurer la confirmabilité des données de même que la piste de vérification qui faisait partie du devis de recherche. Cette piste comprend les données brutes, les produits de réduction et d'analyse des données et un journal des décisions et intentions reliées au processus ainsi que les questions soulevées tout au long de l'étude. La taille réduite de l'échantillon (19 participantes) est une limite puisque cela ne représente qu'un faible pourcentage du nombre total d'infirmières en oncologie du Canada.

\begin{tabular}{|c|c|c|c|c|}
\hline \multicolumn{5}{|c|}{$\begin{array}{l}\text { Tableau 1. Participantes à l'étude- } \\
\text { résumé des données démographiques }\end{array}$} \\
\hline \multicolumn{5}{|c|}{ Tranches d'âge des participantes } \\
\hline \multicolumn{2}{|l|}{ Âge } & \multicolumn{3}{|l|}{ Nombre } \\
\hline \multicolumn{2}{|l|}{$20-30$} & \multicolumn{3}{|l|}{3} \\
\hline \multicolumn{2}{|l|}{$31-40$} & \multicolumn{3}{|l|}{7} \\
\hline \multicolumn{2}{|l|}{$41-50$} & \multicolumn{3}{|l|}{4} \\
\hline \multicolumn{2}{|l|}{$51-60$} & \multicolumn{3}{|l|}{5} \\
\hline \multicolumn{2}{|l|}{ Total } & \multicolumn{3}{|c|}{19 (uniquement des femmes) } \\
\hline \multicolumn{5}{|l|}{ Expérience de travail } \\
\hline Années d'expérience & Min. & Max. & Moyenne & Médiane \\
\hline En tant qu'infirmière & 2 & 33 & 17,16 & 17 \\
\hline $\begin{array}{l}\text { En tant qu'infirmière } \\
\text { en oncologie }\end{array}$ & 2 & 23 & 9,47 & 9 \\
\hline \multicolumn{5}{|l|}{ Expérience clinique } \\
\hline \multicolumn{2}{|l|}{ Milieu de pratique } & \multicolumn{3}{|l|}{ Nombre } \\
\hline \multicolumn{2}{|l|}{ CEuvrant en oncologie } & \multicolumn{3}{|l|}{11} \\
\hline \multicolumn{2}{|l|}{$\begin{array}{l}\text { CEuvrant en dehors de } \\
\text { l'oncologie }\end{array}$} & \multicolumn{3}{|l|}{5} \\
\hline \multicolumn{2}{|l|}{ Pas de réponse } & \multicolumn{3}{|l|}{3} \\
\hline \multicolumn{5}{|l|}{ Niveaux d'instruction } \\
\hline & Inf. & Baccalauréat & $\begin{array}{l}\text { Maîtrise } \\
\text { en } \\
\text { sciences }\end{array}$ & Doctorat \\
\hline Éducation & 6 & 10 & 3 & 0 \\
\hline \multicolumn{5}{|c|}{ Certification de spécialisation (nombre total) } \\
\hline \multicolumn{2}{|l|}{$\begin{array}{l}\text { Détient une certification } \\
\text { en oncologie }\end{array}$} & \multicolumn{3}{|l|}{3} \\
\hline \multicolumn{2}{|c|}{$\begin{array}{l}\text { Ne détient pas de } \\
\text { certification en oncologie }\end{array}$} & \multicolumn{3}{|l|}{15} \\
\hline \multicolumn{2}{|l|}{ Pas de réponse } & \multicolumn{3}{|l|}{1} \\
\hline
\end{tabular}




\section{Résultats}

Les résultats sont organisés selon cinq thèmes majeurs. Les commentaires illustratifs sont présentés dans le tableau 2.

Thème 1: Définir l'usure de compassion. Les répondantes ont fourni des exemples émouvants d'expériences qu'elles associaient à l'UC, et pourtant, elles déclaraient avoir une connaissance limitée de l'UC et se demandaient si oui ou non elles l'avaient éprouvée. Certaines ont dit qu'elles ne connaissaient pas le terme «usure de compassion» ou que si elles l'avaient déjà entendu, elles n'étaient pas sûres de sa signification exacte ni des différences existant entre elle et d'autres expériences comme l'épuisement professionnel.

Aycock et Boyle (2009) ont découvert que l'UC n'a pas de définition précise, ce qui n'empêche pas que «les infirmières en oncologie en saisissent bien le concept» (p. 183, traduction libre). À mesure que nous passions en revue les récits des participantes à l'étude, nous nous sommes aperçus que leurs expériences pourraient nous aider à «définir» l'UC relativement aux infirmières en oncologie. Des attributs communs dans les définitions de l'UC avancées par les infirmières comprenaient un sentiment accablant "que quelque chose n'allait pas », la difficulté qu'elles avaient de se motiver à aller au travail, qu'elles étaient très sensibles d'un point de vue émotionnel et enfin, la fatigue qu'elles ressentaient.

Thème 2: Causes de l'UC. Les causes apparentes de l'UC chez les participantes de l'étude incluaient le manque de soutien, le manque de connaissances sur l'UC et le manque de temps ou l'incapacité de prodiguer des soins optimaux.

\section{Manque de soutien}

La perception d'un manque de soutien semblait associée à l'apparition de l'UC. Les infirmières en oncologie exprimaient le besoin de recevoir du soutien de la part de leurs pairs, de leurs collègues des autres disciplines et de l'administration. De plus, il semblait que la perception continue d'un manque de soutien au fil du temps accentuait le vécu de l’UC.

Beaucoup d'infirmières ont rapporté que de nombreux intervenants avaient de fortes attentes à leur égard, ce qui les amenait à penser qu'elles étaient «tellement fortes» qu'elles n'avaient aucun besoin du soutien d'autrui. En outre, quelques infirmières ont signalé qu'elles hésitaient à admettre qu'elles avaient besoin de soutien, et qu'au contraire il leur fallait projeter une impression de force et d'invincibilité. Cette combinaison de fortes attentes envers elles-mêmes et de la part des autres et de la perception d'un soutien limité semblait constituer un précurseur de l'UC (voir le tableau 2).

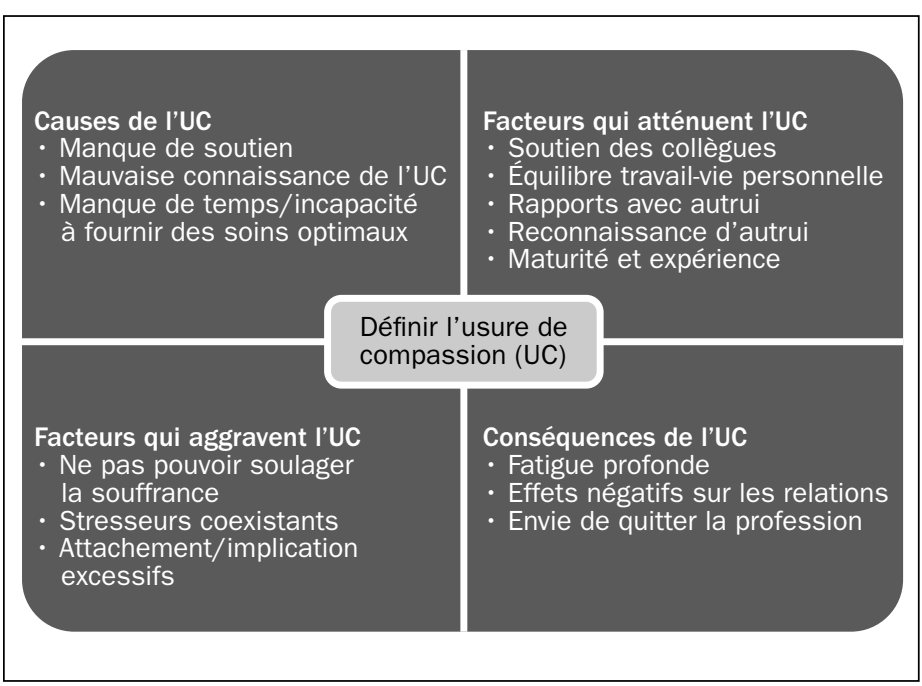

Figure 2. Plan des résultats de l'étude

\section{Manque de connaissances sur l'UC}

Le manque de connaissances sur l'UC était perçu par les infirmières comme une de ses causes. Une répondante a ainsi déclaré: «Il n'y a pas si longtemps que ça que j'ai entendu parler de l'usure de compassion pour la toute première fois.» Une autre a ajouté: «Il nous faut en apprendre davantage sur l'UC afin de nous en protéger.» Certaines répondantes ont précisé que la non-reconnaissance de la survenue possible de ce problème avait pu faire empirer la situation pour elles. Ainsi, lorsqu'elles avaient commencé à éprouver les symptômes de l'UC, plutôt que de croire qu'elles pouvaient éprouver une réaction «naturelle » à leur exposition continue aux souffrances d'autrui, elles se sont mises à penser que c'était chez elles que quelque chose n'allait pas. Les participantes ont signalé que si elles avaient été mieux renseignées au sujet de l'UC et avaient pu prévoir que cette dernière pouvait perturber leur carrière à un moment ou à un autre, elles auraient pu prendre des mesures préventives afin de s'en occuper dès qu'elle posait problème plutôt que d'attendre jusqu'à ce qu'elles ressentent les effets débilitants de l'UC avancée.

\section{Manque de temps/incapacité de prodiguer des soins optimaux}

Beaucoup de répondantes ont fait état d'occasions où il leur avait été impossible de prodiguer aux patients des soins parfaits comme autant d'expériences qui précipitaient la survenue de l'UC. Une infirmière s'est ainsi exprimée: «Il m'arrive parfois de pleurer lorsque je quitte le travail parce que je sens que je n'ai pas pu fournir la qualité de soins que je voulais fournir.» Des soins de première qualité exigent que les infirmières aient le temps de tisser des liens avec les patients et leur famille. Elles ont remarqué qu'elles ont dorénavant moins de temps pour développer cette relation à cause des changements apportés à la charge de travail et à l'organisation de la prestation des soins.

Thème 3: Facteurs aggravant l'UC. Les répondantes ont mentionné divers facteurs qui aggravaient l'UC, notamment les suivants: être incapable de soulager la souffrance, stress physiques et émotionnels coexistants et enfin, engagement et attachement émotionnels excessifs.

\section{Être incapable de soulager la souffrance}

Quand les infirmières sentaient qu'il n'y avait rien qu'elles puissent faire pour aider les patients qui souffraient, l'UC augmentait. Elles décrivaient «l'impuissance» qu'elles ressentaient alors. De nombreuses infirmières avaient choisi les soins infirmiers en oncologie parce qu'elles désiraient «aider les personnes qui avaient vraiment besoin de leur aide». Lorsqu'elles s'apercevaient qu'elles étaient incapables d'aider, elles ressentaient une dissonance qui faisait empirer l'UC.

Stress physiques et émotionnels coexistant en dehors du travail

Dans certains cas, les infirmières décrivaient d'autres difficultés de nature physique et émotionnelle avec lesquelles elles étaient aux prises en dehors du travail et qui faisaient empirer l'UC. Par exemple, une infirmière a décrit la manière dont ses situations de santé personnelles la rendaient plus vulnérable à l'UC dans le cadre de son travail. Une autre répondante établissait également des rapports entre les stresseurs de sa vie privée et l'UC qu'elle éprouvait au travail. Une infirmière a reconnu que la combinaison des stresseurs de la vie personnelle et de la vie professionnelle entraînait des problèmes de santé personnels qui exigeaient une intervention afin d'empêcher «l'usure de compassion caractérisée».

\section{Engagement et attachement émotionnels excessifs}

Bien que la plupart des répondantes aient constaté qu'une partie de leur satisfaction envers leur carrière provenait de l'établissement de liens émotionnels étroits avec les patients et leurs proches, leurs récits renfermaient également des descriptions d'occasions où elles 
éprouvaient un attachement émotionnel «excessif». Les répondantes en concluaient que ces situations pouvaient avoir contribué à leurs épisodes d'UC.

Thème 4: conséquences de l'UC. Les conséquences de l'UC comprenaient entre autres une profonde fatigue, des effets négatifs sur les relations personnelles et l'envie chez certaines infirmières de quitter la profession.

\section{Profonde fatigue psychique et physique}

Nombreuses étaient celles qui rapportaient que la fatigue était le premier signe que quelque chose clochait. La fatigue était profonde, progressive et se traduisait par une lassitude physique et émotionnelle. Une infirmière en oncologie a signalé que la fatigue déclenchait une vague de fatigue encore plus forte jusqu'à ce qu'elle ne vive plus qu'un «cycle de fatigue», pour reprendre ses propres mots. La fatigue profonde incluait des réactions aussi bien physi- ques qu'émotionnelles telles que «une lourdeur au niveau de la poitrine», «des nœuds dans la gorge», «l'esprit embrouillé» et «devoir se retenir de pleurer». Les infirmières ajoutaient que lorsqu'elles recherchaient une attention médicale pour cette fatigue, aucune cause physiologique ne pouvait être dégagée.

\section{Effets négatifs sur les relations personnelles}

Il semblait que l'UC avait des effets négatifs sur les relations personnelles des infirmières. Une répondante associait son UC à la dissolution de son mariage. Des répondantes soupçonnaient les «distractions» au travail de les priver de l'énergie affective dont elles avaient besoin pour établir et entretenir leurs relations familiales et amicales. Les infirmières atteintes d'UC choisissaient souvent de s'isoler. Elles consacraient moins de temps aux activités de loisir en compagnie d'autrui. Elles signalaient qu'elles devenaient désabusées et qu'elles semblaient se désintéresser de tout, ce qui nuisait à leurs relations.

\section{Tableau 2. Commentaires illustratifs des participantes \\ Définir l'UC \\ - Je n'avais pas tout simplement pas l'énergie nécessaire pour continuer. Je me souviens m'être imaginée en train de franchir les portes et d'aller à la réunion de relève et d'avoir pensé "je ne peux pas le faire», mais d'une façon ou d'une autre, j'y parvenais. C'était parfois une véritable lutte psychologique pour y arriver. Je me sentais abattue par mon travail et j'étais tout simplement incapable de continuer. Tu sais, l'étincelle de la vie s'était éteinte en moi. Je fonds en larmes pour des riens, pour des choses qui ne méritent pas de larmes. \\ - Je ressentais une fatigue accablante dont je ne pouvais pas me débarrasser, quelle que soit la quantité de sommeil que j'obtenais. \\ - Un sentiment d'hypervigilance-où mon pouls est un peu plus élevé, ma pression artérielle aussi sans doute, où je suis «constamment en état d'alerte». S'il se produisait un grand bruit soudain, il est possible que je parte en courant. Est-ce ça l'UC?}

Causes de l'UC

1. Manque de soutien

- J'ai donné du soutien à «mes» familles. Mais me suis-je donné du soutien à moi?? Quelqu'un m'a-t-il soutenue? En regardant en arrière, je ne le crois pas.

- D'une façon ou d'une autre, je me voyais ... «super infirmière». J'étais capable d'en faire plus, de résister plus, d'aider plus... plus, plus, plus et ce, sans aucune aide... J'ai payé un lourd tribut.

2. Manque de connaissances sur l'UC

- L'usure de compassion pèse sur l'unité, mais personne ne peut aborder efficacement ce phénomène. Nous en avons à peine entendu parler. Est-ce quelque chose dont nous devrions nous inquiéter? C'est un peu comme s'il nous était impossible de voir l'incidence potentielle de l'usure de compassion sur notre vie.

3. Manque de temps/incapacité de prodiguer des soins optimaux

- Je n'ai plus autant d'occasions d'entretenir des liens étroits avec mes patients et leurs proches. Je passe plus de temps à «superviser les autres» et moins de temps à faire le travail que j'adorais-prendre soins des gens. C'est pourquoi je me sens plus fatiguée à la fin de la journée.

- Je ne crois pas que je puisse m'échapper de ce brouillard qu'est l'usure de compassion tant que je n'aurai pas l'occasion de tisser de nouveau des liens avec les gens, la raison pour laquelle j'avais choisi d'être infirmière en premier lieu! J'ai besoin de prodiguer les meilleurs soins possibles, mais je n'en ai pas le temps.

Facteurs qui aggravent l'UC

1. Être incapable de soulager la souffrance

- Je trimbalais des sentiments de désespoir et de vide... Le service qui m'avait jadis aidé à découvrir mon pouvoir d'action me remplissait désormais de désespoir, et mon usure de compassion a empiré.

- J'ai choisi les soins infirmiers en cancérologie parce que ces patients ont vraiment besoin d'aide et que je voulais les aider.... C'est quand je ne peux pas le faire-soit parce qu'ils ne m'en donnent pas la possibilité, soit parce qu'ils sont extrêmement malades et souffrent énormément-que je sens que je me trouve peut-être dans la mauvaise spécialité. L'usure de compassion prend le dessus.

2. Stress physiques et émotionnels coexistants

- Je ne peux pas vraiment cerner une situation de soins particulière qui m'ait mise sur le carreau. Peut-être s'agissait-il tout simplement... d'un grand nombre de stresseurs qui se manifestaient tous en même temps.

- Étant donné que mon fils souffrant d'insuffisance cardiaque attendait de pouvoir subir une greffe du cour, que mon mariage courait à l'échec, que ma sœur est décédée d'un cancer des ovaires et que mon frère a eu un accident de la route, il a bien fallu que je prenne un pas de recul et que je traverse mes propres tragédies plutôt que de vivre celles des autres; sinon, mon usure de compassion aurait été des plus graves.

3. Engagement et attachement émotionnels excessifs

- Je commence vraiment à me soucier du sort de certains patients. Je me laisse envahir par leurs émotions. Ainsi, ils me suivent jusqu'à la maison, le soir. Même si j'ai une journée de congé, je n'arrête pas de penser à eux. Cela veut dire que je n'ai pas, dans ma tête, la moindre parcelle libre où je pourrais songer sérieusement aux activités amusantes auxquelles je m'adonnerais volontiers. Parfois, je dois bien l'admettre, je m'attache bien trop pour mon propre bien.

- Quand tu vois les patients mal en point, cela t'affecte énormément. Si tu t'es trop attachée à eux et que tu as fait d'eux un peu ta famille, t'as un sacré problème. 
Envisager l'abandon de la profession

De nombreuses répondantes ont dit explicitement que l'UC les avait amenées à envisager l'abandon des soins infirmiers en oncologie ou même de la profession infirmière. L'une d'elles l'a formulé ainsi: «Je ne sais pas du tout si je peux continuer à faire ça le reste de ma vie active.» Une de ses consœurs a offert le commentaire suivant: «Je sens que je ne fais pas la différence que je voulais faire quand j'avais décidé de choisir la vocation d'infirmière. Peut-être que les soins infirmiers ne sont pas faits pour moi». D'ailleurs, quelques répondantes avaient déjà changé de rôles infirmiers à cause de l'UC.

Thème 5 : Circonstances qui atténuent l'UC. Les répondantes ont dégagé des facteurs qui permettent d'atténuer l'UC dont le soutien des collègues, le juste équilibre travail-vie personnelle, le tissage de liens avec autrui, la reconnaissance d'autrui et enfin, la maturité et l'expérience.

\section{Soutien des collègues}

Quand elles décrivaient ce qui atténuait l’UC, les répondantes employaient souvent des mots et groupes de mots tels que "travail en équipe», «soutien mutuel» et «prendre soin les uns des autres».
Elles mettaient l'accent sur le soutien émotionnel qu'elles recevaient de leurs collègues de travail. Selon les infirmières en oncologie ayant moins d'années d'expérience, l'entraide et l'accompagnement mutuel jouent un rôle important dans l'atténuation de l’UC.

\section{Équilibre travail-vie personnelle}

Trouver un juste équilibre travail-vie personnelle était perçu comme une des stratégies d'atténuation de l'UC. Une répondante a fait ce commentaire: «J'ai appris à mettre de côté une partie de moi-même-à réserver une partie de mon cœur à moi et à ma vie privée.» D'autres soulignaient l'importance de prendre du temps pour se ressourcer lorsqu'elles se sentaient terrassées par le stress lié au travail. Elles ajoutaient qu'en menant une vie pleinement satisfaisante en dehors du travail les aidait à voir les choses dans leur contexte lorsque leur travail devenait trop accablant.

\section{Nouer des liens avec autrui}

L'opportunité d'établir des relations avec les patients et leur famille permettait d'atténuer l'UC. Le tissage de liens avec les patients exigeaient que les infirmières apprennent quels étaient les besoins des patients et qu'elles les satisfassent. Elles affirmaient que ceci alimen-

\section{Conséquences de l'UC}

\section{Profonde fatigue psychique et physique}

- J'ai commencé à me sentir plus faible. La douleur et la souffrance incessantes et le fait de voir les gens mourir m'ont affectée personnellement. Je ne me souvenais pas m'être jamais sentie si fatiguée.

- J'avais perdu cette sensation de plénitude que je ressentais avant quand j'œuvrais dans l'unité. Je ne voulais pas qu'on me confie de défis infirmiers dans l'unité. L'envie n'y était pas du tout. J'étais complètement lessivée.

\section{Effets négatifs sur les relations personnelles}

- Il se peut que mon cour ait été endommagé par l'UC. Il est tellement occupé à manifester de la compassion, qu'il ne reste plus de place à l'intérieur pour une relation [personnelle] authentique?

- Ma fille n'arrête pas de me demander «Qu'est-ce qui ne va pas, maman?» Je sais que c'est mon travail, mais je ne le lui dis pas.

3. Envisager l'abandon de la profession

- Pour sûr que j'avais l'impression que ce n'était pas la bonne carrière pour moi. Je crois bien que je devrais me diriger vers la vente immobilière ou quelque chose d'autre juste pour laisser derrière moi la douleur que j'ai ressentie à la vue de tant de tristesse.

- J'ai fini par changer d'emploi, mais mon nouveau poste concernait également des patients en oncologie. Je suis donc encore dans mon domaine des soins infirmiers, mais j'ai pris mes distances vis-à-vis de l'intimité associée aux soins de chevet. Ça m'a aidée à m'adapter.

\section{Circonstances qui atténuent l'UC}

1. Soutien des collègues

- Je travaille au sein d'une merveilleuse équipe et nous prenons soin les uns des autres. Nous travaillons dur ensemble et quand nous avons un cas particulièrement pénible, c'est aussi ensemble que nous faisons notre deuil. Cela fait toute la différence.

- Mes amies au travail m'aident de temps à temps à porter ce qui me pèse, tout comme je les aide à porter le joug de l'usure de compassion quand il devient trop lourd.

2. Équilibre travail-vie personnelle

- Quand j'ai commencé à sentir que cela me dépassait, j'ai pris un petit pas de recul. Pour être efficace au travail, il faut que je m'assure de garder mon autre vie intacte. Le secret, c'est l'équilibre.

3. Nouer des liens avec autrui

- J'adore rire et m'amuser. Je fais tous les efforts pour que ce soit comme ca-et je m'en sens mieux. J'aime mes amis. Prendre le temps de partager des choses avec eux est essentiel pour moi. Grâce à eux, tout va mieux.

- Établir des relations empreintes de bienveillance avec les gens que je soigne. Cela aggrave l'UC et cela l'atténue aussi.

4. Reconnaissance d'autrui

- Le vécu était épuisant et décourageant quelquefois, mais chaque petite victoire le long du chemin, et chaque lueur d'amélioration l'emportent rapidement sur la fatigue liée au vécu.

- De la voir sourire ... et d'atteindre les objectifs que nous avions fixés en collaboration, rendait la fatigue insignifiante. Ma sollicitude était reconnue et appréciée.

5. Maturité et expérience

- Une fois que j'ai été capable de renoncer à l'image féerique que je m'étais donnée de l'infirmière qui pouvait débarquer et réparer tous les problèmes pour autrui, je me suis trouvée libre en quelque sorte de faire de mon mieux et de ne pas en exiger davantage. Il $y$ a des gens qui souffrent alors qu'ils ne le méritent pas. Mais je sais que je peux améliorer un peu le degré de confort de n'importe quelle personne même de celle dont les souffrances sont persistantes et profondes.

- L'usure de compassion se porte sur les épaules, non pas en tant que fardeau qui vient vous clouer au sol, mais comme rappel du grand privilège qu'a l'infirmière en oncologie d'être témoin du courage et de l'amour de ses patients et de leurs proches.

- Quand le joug de l'usure de compassion devient un peu trop lourd, je reconnais sa présence et je me rappelle comment j'en suis arrivée à le porter, comment il m'aide à garder les pieds sur terre et à progresser. 
tait des relations positives les remplissant de satisfaction. Une infirmière a parlé du rôle joué par le tissage de liens amicaux tandis qu'elle s'efforçait de composer avec les stresseurs au travail. Il semblait également que les liens tissés en dehors du travail aidaient à minimiser et peut-être même à empêcher l'apparition de l'UC chez certaines.

\section{Reconnaissance d'autrui}

Les infirmières rapportaient que la reconnaissance reçue des patients, des membres de leur famille, des consœurs et des administrateurs atténuaient l'UC. Une infirmière a fait état d'une très longue et difficile implication auprès d'une patiente. L'infirmière décrivait qu'elle défendait constamment les intérêts de cette patiente et qu'elle ne cessait de penser à la manière dont elle pourrait lui prodiguer les soins dont elle avait besoin. Son dévouement lui a mérité la reconnaissance de la patiente et de ses collègues, une expérience qu'elle a qualifiée d'exaltante. La reconnaissance peut prendre maintes formes. Selon les infirmières, les cartes de remerciement, les notes d'appréciation ou même un sourire sincère sont autant de moyens de faire disparaître leur épuisement.

\section{Maturité et expérience}

Les participants constataient qu'en acquérant de l'expérience au travail-et en assimilant les leçons que cela suppose-elles étaient plus susceptibles de repousser l'UC. Il se peut qu'elles aient appris, grâce à leur expérience croissante, des interventions et approches particulières leur permettant d'aider les souffrants. Lorsqu'elles avaient l'occasion d'intervenir et d'atténuer la souffrance, elles étaient moins susceptibles d'éprouver l'UC.

Les infirmières rapportaient que l'expérience leur permettait d'acquérir une perspective sur la vie qui réduisait leur vulnérabilité face à l'UC. Une fois qu'elles acceptaient que la vie n'est pas toujours juste et qu'elles cessaient de s'attendre à ce qu'elle le soit, elles pouvaient progresser et faire tout leur possible en vue d'aider leurs patients. Une infirmière en oncologie chevronnée était même de l'avis que l'UC pouvait s'avérer positive. À ses dires, l'épuisement émotionnel qu'elle reconnaissait parfois ressentir lui rappelait le rôle privilégié qui était le sien. Cette infirmière expliquait qu'elle en était arrivée à cette perspective sur l'UC après avoir atteint sa pleine maturité professionnelle.

\section{Discussion}

Le modèle du processus de stress (Aneshensel et al., 1995) est d'une grande utilité pour approfondir notre compréhension des thèmes et pour fournir le fondement qui facilitera l'élaboration des implications pour la pratique.

\section{Stresseurs primaires}

Plusieurs stresseurs primaires décrits par les participantes semblaient entretenir des rapports directs avec des interactions de prestation de soins. À titre d'exemple, l'exposition aux souffrances des patients est un stresseur primaire. Les infirmières en oncologie indiquaient qu'elles avaient des interactions quasiment journalières avec des personnes éprouvant des traumatismes physiques, psychiques et spirituels du fait du processus morbide ou des traitements. Ce contact continuel avec les souffrances humaines, surtout lorsqu'il s'agissait d'individus à qui elles s'identifiaient fortement, était un stresseur primaire. L'incapacité à soulager les souffrances était particulièrement stressante.

\section{Stresseurs secondaires}

Le manque de soutien de la part des collègues et des administrateurs constituait un stresseur secondaire pour les infirmières en oncologie ayant participé à l'étude. Les autres stresseurs secondaires incluaient des circonstances de leur vie personnelle telles que des problèmes avec leurs enfants, des relations maritales difficiles, d'autres responsabilités en dehors du travail comme devoir prendre soin de parents âgés ou composer avec leurs propres problèmes de santé. Tous les facteurs qui précèdent ont été identifiés à titre de stresseurs secondaires pouvant contribuer à la survenue de l'UC.

\section{Conditions médiatrices}

Alors que le manque de soutien peut être un stresseur secondaire, le soutien lorsqu'il est fourni, peut constituer une condition médiatrice qui aide à atténuer l'UC. L'expérience et la maturité chez l'infirmière en oncologie ont été décrites comme étant des conditions qui atténuaient les stresseurs et pouvaient aider les infirmières à composer plus efficacement avec les situations stressantes. Dans certains cas, l'expérience et la maturité acquises par les infirmières leur permettaient de mettre les choses en perspective et de voir sous un jour positif leurs interactions avec les patients atteints de cancer. Selon les répondantes, l'existence de marques de reconnaissance appropriées pour leur travail constituait également un facteur atténuant les effets des stresseurs sur leur personne.

\section{Facteurs généraux et contextuels}

La durée de la trajectoire de soins, l'étroitesse de leurs relations avec les patients et les proches, l'intensité de la maladie et des thérapies font toutes partie du contexte dans lequel œuvrent les infirmières en oncologie et peuvent avoir une incidence sur le niveau de stress qu'elles éprouvent et la survenue possible de l'UC. Dans plusieurs de leurs récits, les infirmières indiquaient que l'UC s'était déclenchée après qu'elles eurent soigné des patients ayant fini par décéder.

\section{Résultats}

Les répondantes ont toutes signalé qu'elles avaient pris diverses mesures afin de composer avec l'UC. L'une d'entre elles a changé d'emploi pour adopter un rôle infirmier lui permettant de voir moins de souffrance. D'autres infirmières ont pris de la distance vis-à-vis d'un aspect ou d'un autre de l'intensité de leurs relations avec les patients. Le changement d'attitude est une des autres conséquences consignées par quelques-unes des répondantes. Elles se sont mises à accepter le fait que la vie n'est pas juste et ont ainsi pu composer plus efficacement avec la souffrance. Certaines en sont venues à voir dans leur travail un élément de leur vie qui rendait celle-ci pleinement satisfaisante, ce que les aidait à éviter l'UC.

\section{Interventions possibles en vue d'atténuer l'UC}

\section{Mieux faire connaître l'UC}

On constate un manque de formation et de connaissances à propos de l'UC. Il convient de fournir davantage de connaissances sur tous les aspects de l'UC dans le cadre de la formation d'orientation et de la formation continue. Il est essentiel que les infirmières en oncologie sachent qu'elles risquent d'éprouver l'UC et de promouvoir la reconnaissance précoce de cette condition chez elles-mêmes et chez les autres.

\section{Promotion du travail en équipe et du soutien entre collègues}

Le soutien entre collègues atténue l'UC tandis que le manque de soutien exacerbe les risques. Beaucoup des participantes qui éprouvaient l'UC mentionnaient qu'elles se sentaient «seules». Il est recommandé de mettre en place des politiques et programmes officiels promouvant le travail en équipe et le soutien entre soignants. On pourra ainsi avoir, entre autres, des programmes de modèles d'identification, de précepteurs ou de mentors ou bien favoriser la formation d'équipes interdisciplinaires afin de fournir un soutien mutuel entre collègues par le biais de récapitulations ou de discussions des cas pénibles.

\section{Développer une philosophie «il y a toujours quelque chose de plus qu'on puisse faire»}

Les infirmières éprouvaient l'UC lorsqu'elles se sentaient impuissantes à soulager la souffrance. Elles étaient nombreuses à être 
mécontentes de la qualité des soins qu'elles dispensaient et à se demander si elles «en faisaient assez». Des sentiments de «ne pas pouvoir répondre aux besoins des patients» et de «désespoir» paraissaient être des facteurs communs précipitant l'UC. Les formateurs et les administrateurs doivent donc cultiver parmi le personnel la croyance selon laquelle il y a toujours quelque chose qu'il puisse faire pour diminuer la souffrance. Cette philosophie de soins pourrait habiliter les membres du personnel à faire preuve de créativité et à s'unir en vue d'essayer de soulager la souffrance. En agissant et en refusant d'abandonner la partie, les infirmières pourraient atténuer le sentiment de désespoir qui, d'après elles, les avait menées à l’UC.

\section{Discuter de ce qui constitue un} attachement émotionnel approprié

L'attachement émotionnel aux patients et aux proches est une considération de première importance. Alors que, dans certains cas, il génère un sentiment de satisfaction et donne un sens à leur vie, dans d'autres, il semble aboutir à l'UC. Il est nécessaire d'aborder la question de l'attachement émotionnel lors de l'orientation et du perfectionnement professionnel continu. Il constitue également un sujet important pour les recherches à venir.

\section{Références}

Abendroth, M., \& Flannery, J. (2006). Predicting the risk of compassion fatigue. Journal of Hospice Palliative Care Nursing, 8(6), 346-356.

Absolon, P., \& Krueger, C. (2008). Interventions to manage compassion fatigue in oncology nurses. Oncology Nursing Forum, 35(3), 500.

Adams, R., Figley, C., \& Bascarino, J. (2008). The compassion fatigue scale: Its use with social workers following urban disaster. Research on Social Work Practice, 18(3), 238-250.

Aneshensel, C., Pearlin, L. Mullan, J., Zarit, S., \& Whitlatch, C. (1995). Profiles in caregiving: The unexpected career. San Diego: Academic Press.

Association canadienne des infirmières en oncologie. (n.d.). Roles in oncology. Retrieved from http://www.cano-acio.ca/roles_french/ ?lid $=5$

Aycock, N., \& Boyle, D. (2009). Interventions to manage compassion fatigue in oncology nursing. Clinical Journal of Oncology Nursing, 13(2), 183-192.

Aycock, N., \& Boyle, D. (2008). Overcoming compassion fatigue. Oncology Nursing Forum, 35(3), 524.

Badger, K., Royse, D., \& Craig, C. (2008). Hospital social workers and indirect trauma exposure: An exploratory study of contributing factors. Health and Social Work, 33(1),63-71.

Benoit, L., McCarthy, M., Veach, P., \& LeRoy, B. (2007). When you care enough to do your very best: Genetic counselor experiences of compassion fatigue. Journal of Genetic Counseling, 16(3), 299-313.

Bush, N. (2009). Compassion fatigue: Are you at risk? Oncology Nursing Forum, 36(1), 24-28.

Costa, D. (2005). Compassion fatigue: Self-care skills for practitioners. Occupational Therapy Practice, 10(20),13-18.

Davis, J. (1996). Sadness, tragedy and mass disaster in Oklahoma City: Providing critical incident stress debriefings to a community in crisis. Accident Emergency Nursing, 4, 59-64.

Ferrell, B., \& Coyle, N. (2008). The nature of suffering and the goals of nursing. Oncology Nursing Forum, 35(2), 241-248.

Figley, C. (1995). Compassion fatigue. New York: Brunner/Mazel.

Joinson, C. (1992). Coping with compassion fatigue. Nursing, 92(22), 116-121.

LaRowe, K. (2005). Transforming compassion fatigue into flow. Boston: Acanthus.

Larson, D. (1993). The helper's journey: Working with people facing grief, loss, and life-threatening illness. Champaign, IL: Research Press.

Lincoln, Y., \& Guba, E. (1985). Naturalist Inquiry. Newbury Park, CA: Sage.

\section{Conclusion}

Cette étude fournit des connaissances sur le vécu de l'UC parmi les infirmières canadiennes œuvrant en oncologie clinique. Elle a permis de dégager une définition préliminaire de ce qu'est l'UC pour cette catégorie de soignants et présente une discussion des facteurs pouvant causer, aggraver et/ou atténuer l'UC ainsi que les conséquences de l'UC au sein de ce groupe. L'analyse des résultats effectuée à l'aide du modèle de processus de stress a permis de produire des recommandations pour la pratique qui incluaient instruire les infirmières en matière d'UC, structurer le soutien entre collègues, développer une philosophie des soins qui tende à réduire le sentiment de désespoir et enfin, discuter ouvertement des enjeux relatifs à l'implication émotionnelle auprès des patients et des proches.

\section{Remerciements}

Cette étude a été financée par une subvention pour chercheur chevronné 2008 de l'Association canadienne des infirmières en oncologie (ACIO).

Loiselle, C., \& Profetto-McGrath, J. (2004). Canadian essentials of nursing research. NY: Lippincott Williams \& Wilkins.

Magraith, K., Benson, J., \& Yunti, N. (2005). Compassion fatigue and burnout. Australian Family Physician, 34(6), 497-498.

Maslach, C., Jackson, S., \& Leiter, M. (1996). Maslach burnout inventory manual. PaIo Alto, CA: Consulting Psychologists Press.

McMullen, L. (2007). Compassion fatigue. Oncology Nursing Forum, 34(2), 491-492.

Munroe, J. (1995). Preventing compassion fatigue: A team treatment model. In Compassion fatigue: Coping with secondary traumatic stress disorder in those who treat the traumatized. C. Figley (Ed.). New York: Brunner/Mazel.

Perry, B. (2009). More moments in time: Images of exemplary care. Edmonton, AB: AU Press.

Pfifferling, J., \& Gilley, K. (1999). Overcoming compassion fatigue. Family practice management, 17(3), 36-42.

Oiler, C. (1986). Phenomenology: The method. In P. Munhall \& C. Oiler (Eds.), Nursing research: A qualitative perspective. Norwalk, CT: Appleton-Century-Crofts.

Pickett, M., Brennan, A., Greenberg, H., Licht, L., \& Worrell, J. (1994). Use of debriefing techniques to prevent compassion fatigue in research teams. Nursing Research,43(4), 250-252.

Pierce, B., Dougherty, E., Panzarella, T., Le, L., et al. (2007). Staff stress, work satisfaction, and death attitudes on an oncology palliative care unit, and on a medical and radiation oncology inpatient unit. Journal of Palliative Care, 23(1), 32-42.

Polit, D., \& Hungler, B. (2001). Essentials of nursing research: Methods, appraisal and utilization (5th ed.). New York, N.Y.: Lippincott Williams \& Wilkins.

Robinson, J., Clements, K., \& Land, C. (2003). Workplace stress among psychiatric nurses. Journal of Psychosocial Nursing and Mental Health Services, 41(4), 32-44.

Sabo, B. (2006). Compassion fatigue and nursing work: Can we accurately capture the consequences of caring work? International Journal of Nursing Practice, 12(3), 136-142.

Sabo B. (2008). Are you at risk for compassion fatigue? International Journal of Nursing Practice, 13(3), 136-142.

Stebnicki, M. (2002). Stress and grief reactions among rehabilitation professionals: Dealing effectively with empathy fatigue. Journal of Rehabilitation, 66(1), 23-30.

van Manen, M. (1990). Researching lived experience: Human science for an action sensitive pedagogy. London, ON: Althouse. 